\title{
Unstable Manifolds of Continuous Self-Mappings
}

\author{
Tianxiu Lu ${ }^{1,2}$ and Peiyong $\mathrm{Zhu}^{1}$ \\ ${ }^{1}$ School of Mathematics Sciences, University of Electronic Science and Technology of China, Chengdu 611731, China \\ ${ }^{2}$ Department of Mathematics, Sichuan University of Science and Engineering, Zigong 643000, China
}

Correspondence should be addressed to Tianxiu Lu; lubeeltx@163.com

Received 5 March 2013; Revised 16 May 2013; Accepted 23 May 2013

Academic Editor: Tao Xiang

Copyright (C) 2013 T. Lu and P. Zhu. This is an open access article distributed under the Creative Commons Attribution License, which permits unrestricted use, distribution, and reproduction in any medium, provided the original work is properly cited.

\begin{abstract}
Unstable manifolds of continuous self-mappings on completely densely ordered linear ordered topological spaces (CDOLOTS) are discussed. Let $f$ be a continuous self-map. First, the interval with endpoints of two adjacent fixed points is contained in the unilateral unstable manifold of one of the endpoints. Then, by using the above conclusion, we prove that periodic points of $f$ not belong to the unstable manifold of their iteration points of $f^{n}$ (for some $n \in \mathbb{N}$ ), unless the iteration points are themselves.
\end{abstract}

\section{Introduction}

The complexity of a dynamical system is a central topic of research since the introduction of the term of chaos in 1975 by Li and Yorke [1], known as Li-Yorke chaos today. As concepts relate to chaotic, generalized periodic points are intensively discussed. Throughout this paper, the sets of fixed points, periodic points, $\omega$-limit points, and nonwandering points of a system $(X, f)$ are denoted by $\operatorname{Fix}(f), \operatorname{Per}(f), \omega(x, f)$, and $\Omega(f)$, respectively.

Considering a continuous self-map $f$ on a compact interval $I \subset \mathbb{R}$, Block [2] proved the following results.

(1-1) If $f$ has finitely many periodic points, then the period of each periodic point is a power of 2 .

(1-2) If $\Omega(f)$ is finite, then $\Omega(f)=\operatorname{Per}(f)$.

Based on Block's results, Xiong [3] obtained that

(1-3) $\Omega(f)=\operatorname{Per}(f)$ if and only if $\operatorname{Per}(f)$ is closed,

(1-4) if $\operatorname{Per}(f)$ is closed, then for every point $x$ in $I, \omega(x$, f) $\subset \operatorname{Per}(f)$.

In 2002, Ding and Nadler [4] showed that the invariant set of an $\varepsilon$-contractive map $f$ on a compact metric space $X$ is the same as the set of periodic points of $f$. Furthermore, the set of periodic points of $f$ is finite and, only assuming that $X$ is locally compact, there is at most one periodic point in each component of $X$. Forti et al. [5] gave an example of a triangular map of the unite square, $F(x, y)=\left(f(x), g_{x}(y)\right)$, possessing periodic orbits of all periods and such that no infinite $\omega$-limit set of $F$ contains a periodic point. Moreover, Forti show that there is a triangular map $F$ of type $2^{\infty}$ monotone on the fibres such that any recurrent point of $F$ is uniformly recurrent. And $F$ restricted to the set of its recurrent point is chaotic in the sense of $\mathrm{Li}$ and Yorke. Mai and Shao [6] obtained a structure theorem of graph maps without periodic points, which states that any graph map without periodic points must be topologically conjugate to one of the described class. Recently, Abbas and Rhoades [7] proved that some fixed point theorems in cone metric spaces gave the fact that in a cone with only a partial ordering, the continuous maps have no nontrivial periodic points.

However, the research of generalized periodic points on topological space is very few. The current paper studies continuous self-maps on CDOLOTS. To characterize the properties of continuous self-maps, it is necessary to study unstable manifolds on CDOLOTS. The conclusions in this paper is the generalization of the ones on real line (see $[2,8])$. Furthermore, this paper gave a counterexample to examine that a condition of Theorem 8 is indispensable.

\section{Preliminaries}

Throughout this paper, the order relationship of linear ordered set $X$ is denoted by " $<$ ". And $a \preceq b$ denotes " $a<b$ or $a=b " . \mathcal{N}(x)$ is the system of all open neighborhoods 
of a point $x$. Definitions and notations of intervals in $X$ are similar to the real line. Infinite intervals are denoted by $(\leftarrow, a],[b, \rightarrow)$, and $(\leftarrow, \rightarrow)$. If $(a, b)$ is empty, $a$ will be called for immediate predecessor of $b$ and $b$ will be called for immediate successor of $a$. If there exists an element $m \in X$ such that $x \preceq m$ for all $x \in X, m$ will be called for the largest element of $X$ and denoted by $\operatorname{End}\{X\}$. If there exists an element $m \in X$ such that $m \preceq x$ for all $x \in X, m$ will be called for smallest element of $X$ and denoted by $\operatorname{Ah}\{X\}$. If $X$ is a topological space with the subbase $\{(\leftarrow, b),(a, \rightarrow): a, b \in X\}$, then $X$ is called a linear ordered topological space. We say that $X$ is densely ordered if whenever $a, b \in X$ with $a \prec b$, there is an element $c \in X$ such that $a \prec c \prec b$. We say that $X$ is complete if every nonempty set with upper bound has a least upper bound in $X$. A space $X$ is called a completely densely ordered linear ordered topological space (CDOLOTS), if it is a linear ordered topological space and its order relation is complete and densely ordered. In Munkres [9], there are some examples of CDOLOTS which are different from real line. A separation of $X$ is a pair $U, V$ of disjoint nonempty open subsets of $X$ whose union is $X$. The space $X$ is said to be connected if there does not exist a separation of $X$. According to [9], if $X$ be a CDOLOTS, then $X$ is connected, and so are integers and rays in $X$. A connected set is an interval if it includes more than one point. The definitions of other basic concepts (e.g., continuous self-mapping, periodic point, and periodic orbit) are as usual (see [9-11]).

Let $f: X \rightarrow X$ be a continuous map. Then the image of a closed interval in $X$ is a closed interval. Let $p \in \operatorname{Per}(f)$. The unstable manifold $W^{u}(p, f)$ is defined as follows. Denote $x \in W^{u}(p, f)$ if for any neighborhood $U$ of $x, x \in f^{n}(U)$ for some positive integer $n$. If $p$ is a fixed point of $f$, unilateral unstable manifolds $W^{u}(p, f,+)$ and $W^{u}(p, f,-)$ are defined as follows. Denote $x \in W^{u}(p, f,+)$ if for every interval $K$ with left endpoint $p, x \in f^{n}(K)$ for some positive integer $n$. Denote $x \in W^{u}(p, f,-)$ if for every interval $K$ with right endpoint $p$, $x \in f^{n}(K)$ for some positive integer $n$. By [8], the unstable manifold (or unilateral unstable manifold) of a fixed point on $X$ is connected.

The following property follows easily from the definitions.

Lemma 1. Let $X$ be a CDOLOTS and $f: X \rightarrow X$ a continuous map. If $p$ is a fixed point of $f$, then $W^{u}(p, f)=W^{u}(p$, $f,+) \cup W^{u}(p, f,-)$.

\section{Inclusion Relationship}

Lemma 2. Let $X$ be a general topological space and $Y$ a totally ordered set. $f: X \rightarrow Y$ and $g: X \rightarrow Y$ are continuous maps. Then $\{x \in X: g(x) \prec f(x)\}$ is an open set in $X$.

Proof. Given any $x \in U=\{x \in X: g(x) \prec f(x)\}$, it is clear that $(g(x), \rightarrow) \in \mathcal{N}(f(x))$ and $(\leftarrow, f(x)) \in \mathcal{N}(g(x))$. Now we consider two cases.

Case 1. If $g(x)$ is an immediate predecessor of $f(x)$, by continuity of $f$, it follows that $U_{x}=f^{-1}((g(x), \rightarrow)) \cap$ $g^{-1}((\leftarrow, f(x))) \in \mathcal{N}(x)$. For any $z \in U_{x}$, we have that $f(z) \in(g(x), \rightarrow)$ and $g(z) \in(\leftarrow, f(x))$, then $g(x) \prec$ $f(z), g(z) \prec f(x)$. Noting the fact that $g(x)$ is immediate predecessor of $f(x)$, one has $g(z) \prec f(z)$. This implies that $U_{x} \subset U$.

Case 2. If $g(x)$ is not an immediate predecessor of $f(x)$, then there exist some $y_{0} \in Y$ such that $g(x) \prec y_{0} \prec f(x)$. Clearly, $\left(y_{0}, \rightarrow\right) \in \mathcal{N}(f(x))$ and $\left(\leftarrow, y_{0}\right) \in \mathcal{N}(g(x))$. Thus it is not difficult to check that $V_{x}=f^{-1}\left(\left(y_{0}, \rightarrow\right)\right) \cap g^{-1}\left(\left(\leftarrow, y_{0}\right)\right) \in$ $\mathcal{N}(x)$ and $V_{x} \subset U$.

Summing up Cases 1 and 2, according to the arbitrariness of $x$, it follows that $U$ is an open set in $X$.

In what follows, $X$ is a CDOLOTS and $f: X \rightarrow X$ a continuous map.

Lemma 3. Let $p_{1}, p_{2} \in X$ with $p_{1} \prec p_{2}$. If $\operatorname{Fix}(f) \cap\left(p_{1}, p_{2}\right)=$ $\emptyset$, then one of the following two cases holds:

(i) $x \prec f(x)$ for any $x \in\left(p_{1}, p_{2}\right)$;

(ii) $f(x) \prec x$ for any $x \in\left(p_{1}, p_{2}\right)$.

Proof. Suppose that there exist $x_{1}, x_{2}$ in $\left(p_{1}, p_{2}\right)$ such that $x_{1} \prec f\left(x_{1}\right)$ and $f\left(x_{2}\right) \prec x_{2}$. Define a continuous map $g: X \rightarrow X$ by $g(x)=x$ for any $x \in X$. Let us take

$$
\begin{aligned}
& U=\left\{x \in\left(p_{1}, p_{2}\right): g(x) \prec f(x)\right\}, \\
& V=\left\{x \in\left(p_{1}, p_{2}\right): f(x) \prec g(x)\right\} .
\end{aligned}
$$

Clearly, $x_{1} \in U$ and $x_{2} \in V$. Combining this with Lemma 2, it is not difficult to check that $U$ and $V$ constitute a separation of $\left(p_{1}, p_{2}\right)$, which contradicts the connectivity of $\left(p_{1}, p_{2}\right)$.

Theorem 4. If $p_{1}$ and $p_{2}$ are two adjacent fixed points of $f$ with $p_{1} \prec p_{2}$, then

$$
\left(p_{1}, p_{2}\right) \subset W^{u}\left(p_{1}, f,+\right) \quad \text { or } \quad\left(p_{1}, p_{2}\right) \subset W^{u}\left(p_{2}, f,-\right) \text {. }
$$

Proof. Since $p_{1}$ and $p_{2}$ are two adjacent fixed points of $f$ with $p_{1} \prec p_{2}$, applying Lemma 3 , it follows that one of the following cases holds:

(i) $x \prec f(x)$ for any $x \in\left(p_{1}, p_{2}\right)$;

(ii) $f(x) \prec x$ for any $x \in\left(p_{1}, p_{2}\right)$.

Without loss of generality, we may assume that case (i) holds.

Now we assert that $\left(p_{1}, p_{2}\right) \subset W^{u}\left(p_{1}, f,+\right)$. That is, given any fixed $y \in\left(p_{1}, p_{2}\right)$, for any $z \in\left(p_{1}, \rightarrow\right)$, there exists a $k \in \mathbb{N}$ such that $y \in f^{k}\left(\left[p_{1}, z\right]\right)$. Take $\alpha=\operatorname{End}\left\{f\left(\left[p_{1}, z\right]\right)\right\}$. To prove this, we consider three cases.

Case $1\left(z \in\left(p_{1}, y\right]\right)$. Noting that $x \prec f(x)$ holds for any $x \in$ $\left(p_{1}, p_{2}\right)$ and that $p_{1} \in \operatorname{Fix}(f)$, one has that

$$
\begin{gathered}
z \prec f(z) \preceq \alpha, \\
{\left[p_{1}, z\right] \subset\left[f\left(p_{1}\right), f(z)\right] \subset f\left(\left[p_{1}, z\right]\right) .}
\end{gathered}
$$

(1-1) If $y \prec \alpha$, then $y \in f\left(\left[p_{1}, z\right]\right)$.

(1-2) If $\alpha \prec y$, we have that $y \in f^{k}\left(\left[p_{1}, z\right]\right)$ for some $k \geq 2$. 
In fact, suppose that $y \notin f^{k}\left(\left[p_{1}, z\right]\right)$ holds for any $k \geq 2$, and then

$$
y \prec \operatorname{Ah}\left\{f^{k}\left(\left[p_{1}, z\right]\right)\right\} \quad \text { or } \quad \text { End }\left\{f^{k}\left(\left[p_{1}, z\right]\right)\right\} \prec y .
$$

For any $k \geq 2$, as $\operatorname{Ah}\left\{f^{k}\left(\left[p_{1}, z\right]\right)\right\} \preceq f^{k}\left(p_{1}\right)=p_{1} \prec y$, we have End $\left\{f^{k}\left(\left[p_{1}, z\right]\right)\right\}<y$. Combining this with the fact that $x \prec f(x)$ holds for any $x \in\left(p_{1}, p_{2}\right)$, it follows that

$$
p_{1} \prec z \prec f(z) \prec f^{2}(z) \prec \cdots \prec f^{k}(z) \prec \cdots \prec y \prec p_{2} .
$$

Take $\beta=\sup \left\{f^{k}(z): k \in \mathbb{N}\right\}$. Clearly, $\beta \in\left(p_{1}, p_{2}\right)$. Meanwhile, it is easy to see that

$$
\begin{aligned}
\beta & =\lim _{k \rightarrow \infty} f^{k}(z)=\lim _{k \rightarrow \infty} f\left(f^{k-1}(z)\right) \\
& =f\left(\lim _{k \rightarrow \infty} f^{k-1}(z)\right)=f(\beta) .
\end{aligned}
$$

Case $2\left(z \in\left(y, p_{2}\right)\right)$. Observing that $z \prec f(z)$ and $f\left(p_{1}\right)=p_{1}$, we know that $y \in\left[p_{1}, z\right] \subset\left[f\left(p_{1}\right), f(z)\right] \subset f\left(\left[p_{1}, z\right]\right)$.

Case $3\left(z \in\left(p_{2}, \rightarrow\right)\right)$. Then $y \in\left[p_{1}, p_{2}\right] \subset f\left(\left[p_{1}, p_{2}\right]\right) \subset$ $f\left(\left[p_{1}, z\right]\right)$.

According to Lemma 1 and Theorem 4, Corollary 5 follows immediately.

Corollary 5. If $p_{1}$ and $p_{2}$ are two adjacent fixed points of $f$ with $p_{1} \prec p_{2}$, then

$$
\left(p_{1}, p_{2}\right) \subset W^{u}\left(p_{1}, f\right) \quad \text { or } \quad\left(p_{1}, p_{2}\right) \subset W^{u}\left(p_{2}, f\right) .
$$

\section{Separability}

Lemma 6. Let $K \subset X$ be a nonempty closed interval such that $K \subset f(K)$ or $f(K) \subset K$. Then $\operatorname{Fix}(f) \cap K \neq \emptyset$.

Proof. Without loss of generality, we may assume that $K=$ $[a, b]$.

When $f(K) \subset K$, this holds trivially. It remains to consider the case that $K \subset f(K)$.

Since $K \subset f(K)$, then there exist some $x, y \in K$ such that

$$
f(x)=a \preceq x, \quad y \geq b=f(y) .
$$

(1) If $f(x)=x$ or $f(y)=y$, the conclusion is clear.

(2) If $f(x) \neq x$ and $f(y) \neq y$, then $f(x)<x$ and $y<f(y)$. $(x ; y)$ is an open set with endpoints $x$ and $y$. Then, there exists an element $z$ in $(x ; y)$ such that $f(z)=z$. Otherwise, it contradicts that $[x ; y]$ is a connect set.

Lemma 7. Let $p$ is a fixed point of $f$ and $x \in W^{u}(p, f)$. Then $x \in W^{u}(p, f,+)$ if $p \prec x$ and $x \in W^{u}(p, f,-)$ if $x \prec p$.
Proof. Suppose that $p \prec x$ (the case $x<p$ is similar).

Case 1. If $p$ is the smallest element of $X$, then $K=\left[p, b_{0}\right) \in$ $\mathcal{N}(p)$ for arbitrary $b_{0}$ in $X$. Since $x \in W^{u}(p, f)$, one has $x \in$ $f^{n}(K)$ for some integer $n$. Thus, $x \in W^{u}(p, f,+)$.

Case 2. If $p$ is not the smallest element of $X$, let $p_{1}$ is a fixed point adjacent to $p$ with $p_{1} \prec p$ (or let arbitrary $p_{1}$ in $X$ which satisfied $p_{1} \prec p$ if there are no fixed points which is ahead of $p)$. Since $\left(p_{1}, p\right) \cap \operatorname{Fix}(f)=\phi$, then.

(i) $t \prec f(t)$ for every $t$ in $\left(p_{1}, p\right)$ or (ii) $f(t) \prec t$ for every $t$ in $\left(p_{1}, p\right)$.

And because $p$ is a fixed point of $f$, one has $f^{n}\left(\left(p_{1}, p\right]\right) \subset$ $(\leftarrow, p]$ for every positive integer $n$.

We prove $x \in W^{u}(p, f,+)$ by contradiction.

Suppose $x \notin W^{u}(p, f,+)$, then $x \in W^{u}(p, f,-)$. There exists a positive integer $n_{1}$ such that $x \in f^{n_{1}}\left(\left(p_{1}, p\right]\right) \subset(\leftarrow, p]$. Then $x \preceq p$, a contradiction.

Thus $x \in W^{u}(p, f,+)$.

Theorem 8. If $f$ has finitely many periodic points. $\left\{p_{1}, p_{2}, \ldots, p_{n}\right\}$ is a periodic orbit of $f$ with period $n$. And $p_{i}, p_{j}$ are distinct elements on the periodic orbit. Then $p_{j} \notin W^{u}\left(p_{i}, f^{n}\right)$.

Proof. According to the connectivity of the unstable manifold at a fixed point, it is obvious that $W^{u}\left(p_{i}, f^{n}\right)$ is an interval. By resorting, one can assume that $p_{1} \prec p_{2} \prec \cdots \prec p_{n}$. Use reduction to absurdity.

Suppose there exist $i, j(i \neq j, i, j=1,2, \ldots, n)$ such that $p_{j} \in W^{u}\left(p_{i}, f^{n}\right)$.

For arbitrary $k \in\{1,2, \ldots, n\}$.

If $k=i$, then

$$
p_{j} \in W^{u}\left(p_{k}, f^{n}\right) \cap\left(\left\{p_{1}, p_{2}, \ldots, p_{n}\right\} \backslash\left\{p_{k}\right\}\right) .
$$

If $k \neq i$, then $f^{r}\left(p_{i}\right)=p_{k}$ for some $r(1 \leq r \leq n)$. Since $f^{r}$ is a continuous map, there exists a neighborhood $U$ of $p_{i}$ such that $f^{r}(U) \subset V$ for every neighborhood $V$ of $p_{k}$. Because $p_{j} \in$ $W^{u}\left(p_{i}, f^{n}\right)$, there exists an integer $s$ such that $p_{j} \in f^{n s}(U)$. Then

$$
f^{r}\left(p_{j}\right) \in f^{r}\left(f^{n s}(U)\right)=f^{n s}\left(f^{r}(U)\right) \subset f^{n s}(V) .
$$

One has

$$
f^{r}\left(p_{j}\right) \in W^{u}\left(p_{k}, f^{n}\right) .
$$

Since $f^{r}\left(p_{i}\right)=p_{k}$ and $p_{i} \neq p_{j}$, one has $f^{r}\left(p_{j}\right) \neq p_{k}$. Then

$$
f^{r}\left(p_{j}\right) \in W^{u}\left(p_{k}, f^{n}\right) \cap\left(\left\{p_{1}, p_{2}, \ldots, p_{n}\right\} \backslash\left\{p_{k}\right\}\right) .
$$

Combining with (9) and (12), clearly, for arbitrary $k \in$ $\{1,2, \ldots, n\}, W^{u}\left(p_{k}, f^{n}\right)$ contains at least one element of $\left(\left\{p_{1}, p_{2}, \ldots, p_{n}\right\} \backslash\left\{p_{k}\right\}\right)$. Thus $W^{u}\left(p_{1}, f^{n}\right)$ is an interval which contains $p_{1}$ and $p_{2}$. Similarly, one has

$$
p_{1} \in W^{u}\left(p_{2}, f^{n}\right) \quad \text { or } \quad p_{3} \in W^{u}\left(p_{2}, f^{n}\right) .
$$

Next we will show that $p_{1} \notin W^{u}\left(p_{2}, f^{n}\right)$. 
In fact, by Lemma 7 , if $p_{1} \in W^{u}\left(p_{2}, f^{n}\right)$, then $p_{1} \in$ $W^{u}\left(p_{2}, f^{n},-\right)$ and $p_{2} \in W^{u}\left(p_{1}, f^{n},+\right)$. One has $f^{n m}(x)=p_{2}$ for some integer $m$ and some $x$ in $\left(p_{1}, p_{2}\right)$.

Put

$$
z=\operatorname{Ah}\left\{x \in\left(p_{1}, p_{2}\right): f^{n m}(x)=p_{2}\right\} .
$$

For arbitrary $a$ in $\left(p_{1}, z\right)$, there exists an element $x$ in $[a, z)$ such that $f^{n m}(x) \prec p_{2}$ (otherwise it contradicts the definition of $z)$. Then $f^{n m}([a, z])$ contains an interval of the form $\left[b, p_{2}\right]$. Since $p_{1} \in W^{u}\left(p_{2}, f^{n},-\right)$, for the above interval $\left[b, p_{2}\right]$, there exists a positive integer $s$ such that $p_{1} \in f^{n s}\left(\left[b, p_{2}\right]\right)$. And because $f^{n m}([a, z]) \supset\left[b, p_{2}\right]$, then

$$
p_{1} \in f^{n s}\left(f^{n m}([a, z])\right)=f^{n(s+m)}([a, z]) .
$$

By $f^{n m}(z)=p_{2}$, one has $p_{2} \in f^{n(s+m)}([a, z])$. Then

$$
f^{n(s+m)}([a, z]) \supset\left[p_{1}, p_{2}\right] \supset[a, z] .
$$

So $f$ has a periodic point in $[a, z]$ by Lemma 6. Since $a$ is an arbitrary point with $p_{1} \prec a \prec z$, then $f$ has infinitely many periodic points, a contradiction.

Therefore, by (13), $p_{3} \in W^{u}\left(p_{2}, f^{n}\right)$.

Similar to the above discussion, it follows that

$$
p_{i+1} \in W^{u}\left(p_{i}, f^{n}\right) \quad \text { for } i=1,2, \ldots, n-1 .
$$

In particular, $p_{n} \in W^{u}\left(p_{n-1}, f^{n}\right)$. But $W^{u}\left(p_{n}, f^{n}\right)$ is an interval containing $p_{n}$ and some elements of $\left\{p_{1}, p_{2}, \ldots, p_{n-1}\right\}$, then $p_{n-1} \in W^{u}\left(p_{n}, f^{n}\right)$. By the same argument as the preceding paragraphs, it follows that $f$ has infinitely many periodic points, a contradiction.

We thus conclude that $p_{j} \notin W^{u}\left(p_{i}, f^{n}\right)$.

Remark 9. Since $\mathbb{R}$ is the most natural example of CDOLOTS, Lemma 4 in [8], Lemma 3 in [8], Lemma 6 in [2], and Theorem 8 in [2] are corollaries of Theorem 4, Lemma 6, Lemma 7, and Theorem 8 in this paper, respectively.

Remark 10. Practically, the results of Theorems 4 and 8 are ture for continuous maps on compact intervals. This paper makes it clear that topological structure of spaces has no impact on these results, if the spaces are completely and densely ordered.

Remark 11. The condition " $f$ has finitely many periodic points" in Theorem 8 cannot be removed. We will give an example to show it.

Example 12. Triangular tent map $f:[0,1] \rightarrow[0,1]$ by $f(x)=2 x$ for $0 \leq x \leq 1 / 2$ and $f(x)=2-2 x$ for $1 / 2<x \leq 1$.

As one knows, for every positive integer $n, f$ has periodic points with period $n$. So $f$ has infinitely many periodic points.

According the image of $f^{n}$ for every positive integer $n, f^{n}$ is a periodic function with period $1 / 2^{n}$. $[0,1]$ was divided into $2^{n-1}$ subintervals which have the same length $1 / 2^{n-1}$. That is, $\left[0,1 / 2^{n-1}\right],\left[1 / 2^{n-1}, 2 / 2^{n-1}\right],\left[2 / 2^{n-1}, 3 / 2^{n-1}\right], \ldots,\left[\left(2^{n-1}-1\right) /\right.$ $\left.2^{n-1}, 1\right]$. The image of $f^{n}$ on every subinterval is the same.
The image on the first subinterval $\left[0,1 / 2^{n-1}\right]$ was observed, with $f^{n}$ increasing from 0 to 1 with slope $2^{n}$ on $\left[0,1 / 2^{n}\right]$ and decreasing from 1 to 0 with slope $-2^{n}$ on $\left[1 / 2^{n}, 1 / 2^{n-1}\right]$. So, it is easy to check that there exists a positive integer $n$ such that $f^{n}((a, b)) \supset[0,1]$ for every open integer $(a, b)$ (there $0 \leq a<b \leq 1)$.

Now, we study the 2-periodic orbit $\{2 / 5,4 / 5\}$ of $f$. For every $U \in \mathcal{N}(4 / 5)$, let $U=(a, b)$ (there $0 \leq a<4 / 5<b \leq 1)$. By the previous paragraph, there exists a positive integer $n$ such that $f^{n}(U)=[0,1]$. Then there exists an even $n$ such that $2 / 5 \in f^{n}(U)$. That is, $2 / 5 \in w^{u}\left(4 / 5, f^{2}\right)$.

\section{Acknowledgments}

This project was supported by the Fundamental Research Funds for the Central Universities, the Scientific Research Fund of Sichuan Provincial Education Department (no. 12ZA098), and Artificial Intelligence of Key Laboratory of Sichuan Province (no. 2012RYY04).

\section{References}

[1] T. Y. Li and J. A. Yorke, "Period three implies chaos," The American Mathematical Monthly, vol. 82, no. 10, pp. 985-992, 1975.

[2] L. Block, "Continuous maps of the interval with finite nonwandering set," Transactions of the American Mathematical Society, vol. 240, pp. 221-230, 1978.

[3] J. C. Xiong, "Continuous self-maps of the closed interval whose periodic points form a closed set," Journal of China University of Science and Technology, vol. 11, no. 4, pp. 14-23, 1981.

[4] C. Ding and S. B. Nadler, "The periodic points and the invariant set of an $\varepsilon$-contractive map," Applied Mathematics Letters, vol. 15, no. 7, pp. 793-801, 2002.

[5] G. Forti, L. Paganoni, and J. Smítal, "Triangular maps with all periods and no infinite $\omega$-limit set containing periodic points," Topology and Its Applications, vol. 153, no. 5-6, pp. 818-832, 2005.

[6] J. Mai and S. Shao, "The structure of graph maps without periodic points," Topology and Its Applications, vol. 154, no. 14, pp. 2714-2728, 2007.

[7] M. Abbas and B. E. Rhoades, "Fixed and periodic point results in cone metric spaces," Applied Mathematics Letters, vol. 22, no. 4, pp. 511-515, 2009.

[8] L. Block, "Homoclinic points of mappings of the interval," Proceedings of the American Mathematical Society, vol. 72, no. 3, pp. 576-580, 1978.

[9] J. Munkres, Topology, China Machine Press, 2nd edition, 2004.

[10] J. Nagata, Modern General Topology, North-Holland Publishing, Amsterdam, The Netherlands, 1985.

[11] S. Todorcevic, Trees and Linearly Ordered Set, North-Holland, Amsterdam, The Netherlands, 1984. 


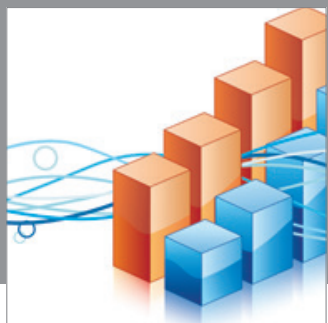

Advances in

Operations Research

mansans

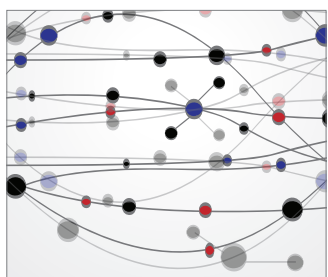

The Scientific World Journal
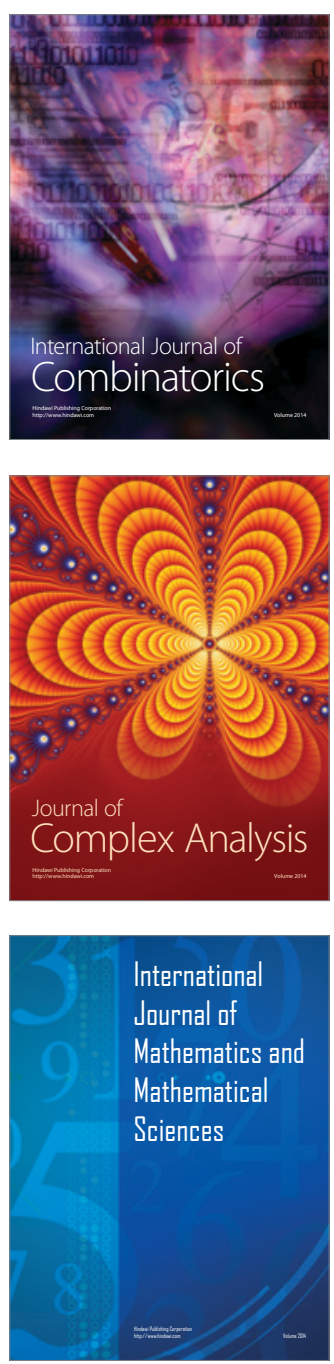
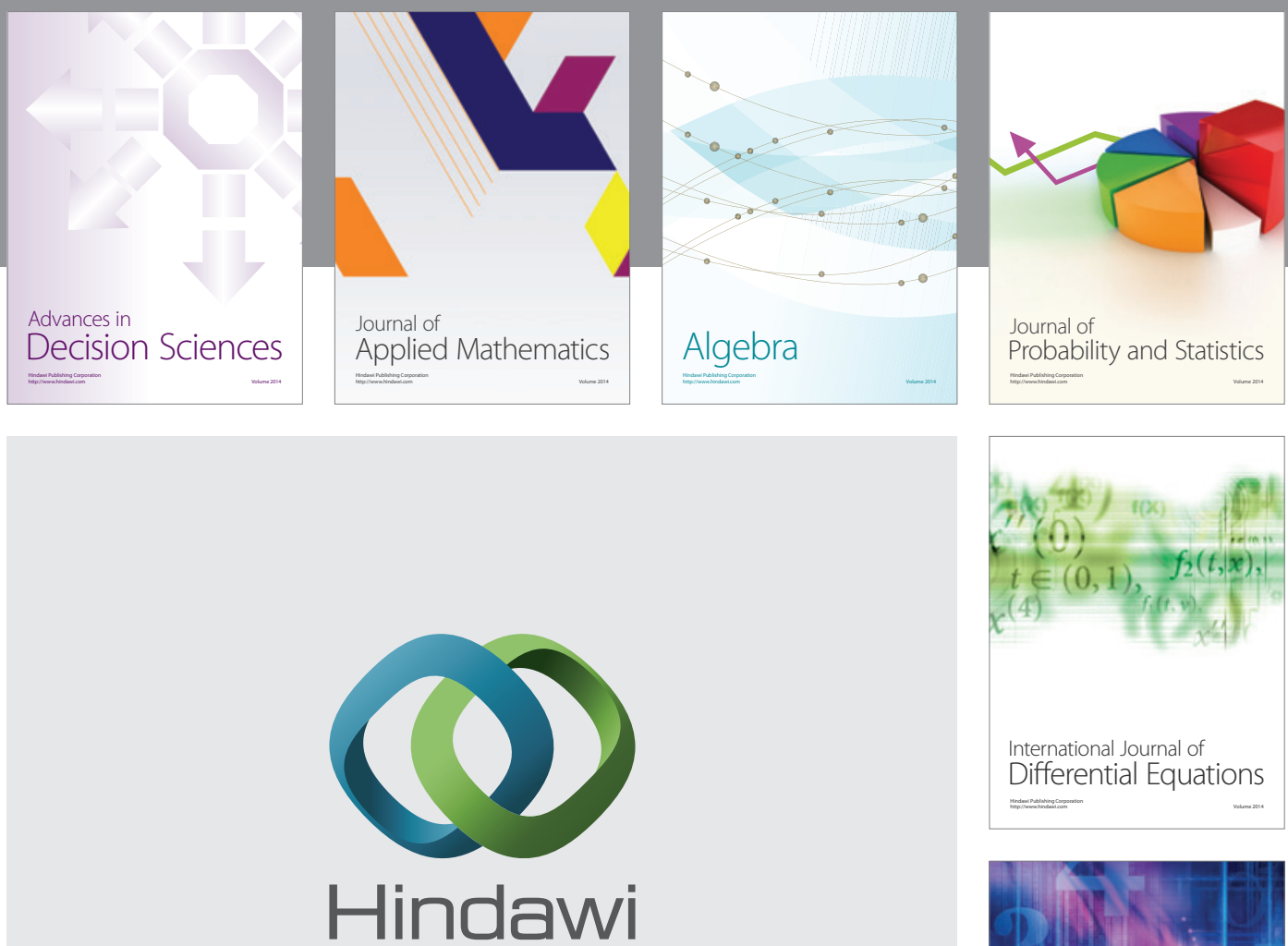

Submit your manuscripts at http://www.hindawi.com
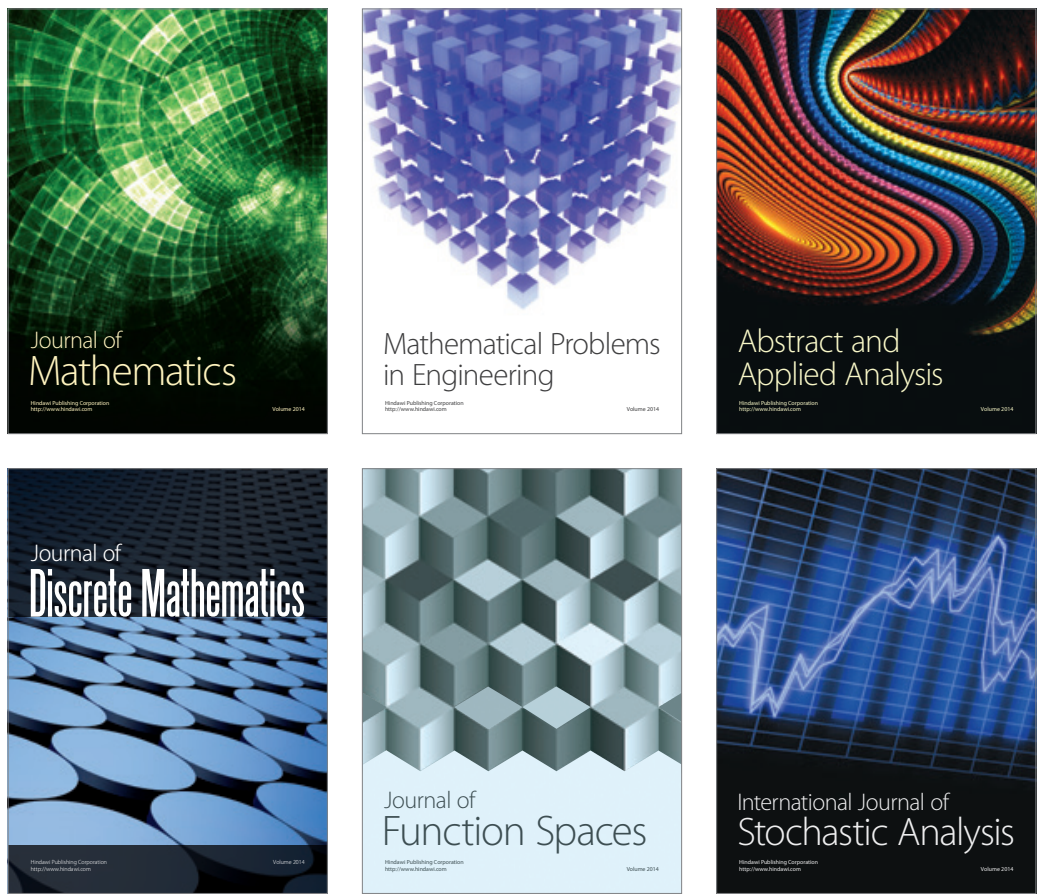

Journal of

Function Spaces

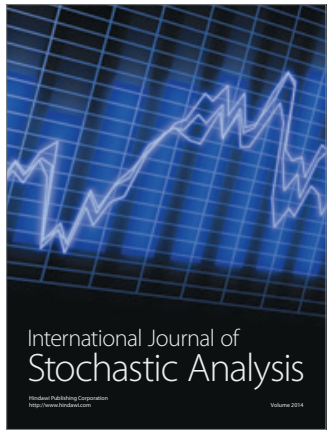

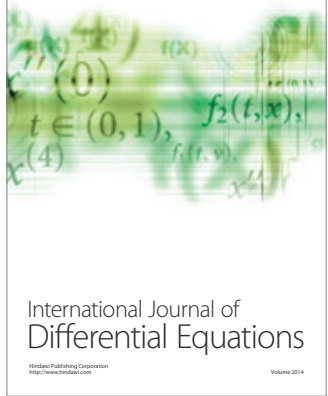
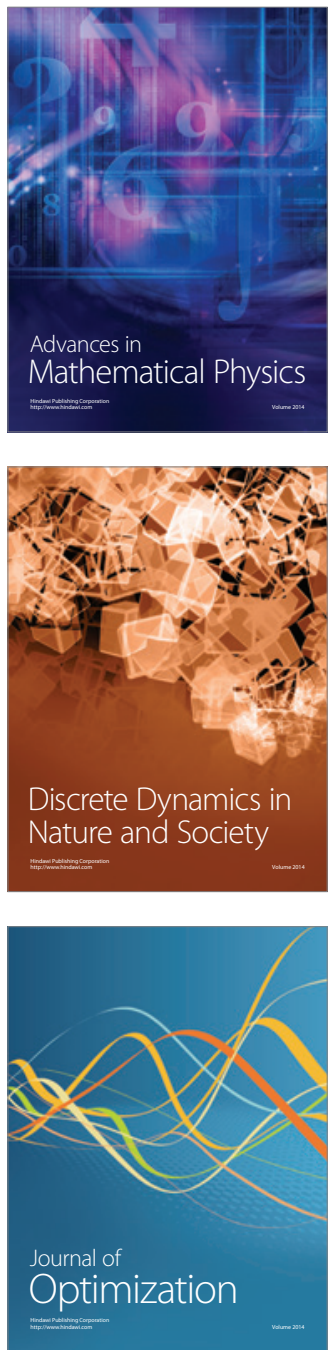